\title{
An inexact optimization model associated with two robust programming approaches for water resources management
}

\author{
W. Li $\cdot$ M. Liu $\cdot$ S. Z. Wu $\cdot$ Y. Xu
}

Received: 29 November 2013/Revised: 27 April 2014/ Accepted: 9 June 2014/Published online: 5 July 2014

(C) Islamic Azad University (IAU) 2014

\begin{abstract}
In this study, an interval fuzzy-robust two-stage stochastic-robust programming (IFRTSRP) model is developed for water resources management under uncertainty. The developed IFRTSRP model incorporates two-stage stochastic programming (TSP), fuzzy robust programming (FRP), interval linear programming (ILP), and stochastic robust optimization (SRO) within a general optimization framework. The IFRTSRP model can not only deal with uncertainties presented as probability distributions, fuzzy membership functions, discrete interval numbers, and their combinations, but also provide an effective linkage between the pre-regulated water resources management policies and the associated economic implications. The IFRTSRP model can also enhance the robustness for the optimization process by delimiting the uncertain decision space through dimensional enlargement of the original fuzzy constraints. Moreover, the IFRTSRP model can evaluate the trade-offs between system economy and stability by incorporating the variability measures on penalty costs into the objective function. The IFRTSRP model is applied to a hypothetical case study of water resources management. The results indicate that reasonable solutions would be generated under different levels of
\end{abstract}

\section{W. Li · M. Liu}

MOE Key Laboratory of Regional Energy and Environmental Systems Optimization, Resources and Environmental Research Academy, North China Electric Power University, Beijing 102206, China

M. Liu

Electric Power Research Institute of State Grid Zhejiang Electric Power Company, Hangzhou 310014, China

S. Z. Wu $(\bowtie) \cdot$ Y. Xu

Chinese Academy for Environmental Planning,

Beijing 100012, China

e-mail:wusz@caep.org.cn $\lambda$ and/or $\omega$ (non-negative weight coefficients); moreover, a higher net system benefit would correspond to lower system stability and higher system failure risk. Thus, the modeling results can be used for generating decision alternatives and thus help the managers to identify desired water allocation policies based on the reasonable consideration of system economy, system stability, and system failure risk.

Keywords Water resources management - Robust . Modeling · Optimization · Uncertainty

\section{Introduction}

Water is one of the most important natural resources, particularly in arid and semi-arid regions. Speedy population growth, rapid socio-economic development, and variational natural conditions have led to increasing reliance on water resources (Wang et al. 2005). In most situations, only surface water resources can hardly satisfy the essential demands of water users due to the low efficiency of water exploration and the insufficiency of water availability; nevertheless, ground water resources can be regarded as a necessary supplement to satisfy the water demands (Hoppe et al. 2004; Olsen et al. 2006; Chenini et al. 2008; Lu et al. 2009). Meanwhile, the increased water demands and the unreliable water supplies have been considered as major barriers to sustainable water resources management. Thus, surface and ground water resources should be conjunctively used to address water crisis issues; moreover, various optimization techniques should be developed to formulate the cost-effective and environment-friendly water allocation schemes and policies. However, in many real-world problems, the achievement of sound strategies is different since water resources management systems are 
complicated with a variety of uncertainties and their interactions. Uncertainties can not only be derived from random nature but also arise from fuzziness and imprecision due to available data deficiencies and biased judgments. For example, water availabilities are influenced by stochastic events, which may fluctuate from time to time; moreover, since they are hard to be precisely determined even with given probabilities, they are also presented as intervals with fuzzy lower and upper bounds (namely "fuzzy boundary intervals"). Some uncertain information, such as benefit and cost coefficients and water allocation targets, may vacillate within a certain discrete intervals.

As a result, a large number of research efforts were undertaken to deal with the above difficulties in water resources management systems through various modeling approaches (Trezos and Yeh 1987; Chang et al. 1996; Feiring et al. 1998; Jairaj and Vedula 2000; Karamouz et al. 2004; Li et al. 2006a; Sethi et al. 2006; Lu et al. 2009; Sadegh et al. 2010; Hu et al. 2012; Amin et al. 2013; Han et al. 2013). Among them, two-stage stochastic programming (TSP) is effective in dealing with problems where an analysis of policy scenarios is desired and when the right-hand side coefficients are random with known probability distributions, and can facilitate the generation of effective management strategies (Maqsood and Huang 2003; Kara and Onut 2010; Noyan 2012). In TSP, an initial decision must be made before the realization of random variables (the first stage), and then a corrective action can be taken after random events have taken place (the second stage); this implies that the second-stage decision can be used to minimize "penalties" that may appear due to any infeasibility (Li and Huang 2009; Liu et al. 2013). TSP has been widely applied for water resources management (Huang and Loucks 2000; Maqsood et al. 2005; Guo and Huang 2009; Shao et al. 2011). However, in water resources management systems, the quality of available information is often not satisfactory enough for establishing probability distributions, and TSP is incapable of dealing with uncertainties as mixtures of vagueness and intervals; moreover, the increased data requirements for specifying the probability distributions of parameters may affect the practical applicability of TSP method. Instead, fuzzy robust programming (FRP), which is based on fuzzy set theory, can effectively reflect fuzzy uncertainties in both left- and right-hand sides coefficients (of the model's constraints) as represented by fuzzy membership functions (Liu et al. 2003; Singh and Dhillon 2008). By delimiting an uncertain decision space through dimensional enlargement of the original fuzzy constraints, FRP can enhance the robustness of the optimization process (Nie et al. 2007; Cai et al. 2009). Interval linear programming (ILP) can effectively tackle uncertainties expressed as discrete intervals. ILP allows uncertainties to be directly communicated into the optimization process and resulting solutions; moreover, it does not lead to more complicated intermediate models and does not require distribution information for model parameters (Xu et al. 2009a). Applications of FRP and ILP have been reported in the field of water resources management (Huang 1996; Tan and Cruz 2004; Li et al. 2009; Guo et al. 2010; Han et al. 2011). Therefore, in water resources management systems, to better account for multiple uncertainties and economic penalties, one potential approach is to incorporate the methods of TSP, FRP and ILP within a general optimization framework, which leads to an interval fuzzyrobust two-stage stochastic programming (IFRTSP) method.

In water resources management systems, in addition to the pursuit in the maximization of net benefit or the minimization of cost, the system stability and water allocation patterns should be considered; however, in the modeling processes, the IFRTSP method can hardly reflect the risk of model feasibility and reliability. Instead, stochastic robust optimization (SRO), one of the stochastic mathematical programming methods, can bring risk aversion into optimization models and find robust solutions (Mulvey and Ruszczynski 1995; Dupačová 1998). In SRO, the uncertain parameters are tackled as random variables with discrete distributions. SRO integrates a goal programming formulation with a scenario-based description of problem data, and can generate a series of solutions that are progressively less sensitive to realizations of problem data from a set of scenarios (Mulvey et al. 1995; Leung et al. 2007; $\mathrm{Xu}$ et al. 2009a). SRO can also help decision makers to quantitatively evaluate the trade-offs between system economy and stability. SRO has been applied to the field of water resources management (Watkins Jr and Mckinney 1997; Xu et al. 2009b; Gaivoronski et al. 2012; Chen et al. 2013). Therefore, in water resources management systems, to better utilize the strengths of different methods and formulate the effective management strategies, SRO method is incorporated within a general IFRTSP framework, which leads to an interval fuzzy-robust two-stage stochastic-robust programming (IFRTSRP) method. Previously, few studies focused on the IFRTSRP method for water resources management under multiple uncertainties.

Therefore, this study aims to develop an optimization model based on the IFRTSRP method for water resources management under uncertainty. As an integration of TSP, FRP, ILP, and SRO, the developed IFRTSRP model can not only deal with multiple uncertainties expressed as probability distributions, fuzzy membership functions, discrete intervals, and their combinations, but also provide an effective linkage between the pre-regulated water resources management policies and the associated economic implications. The IFRTSRP model can enhance the robustness for the optimization process by delimiting the uncertain decision space through dimensional enlargement of the original fuzzy constraints. Moreover, the IFRTSRP model is capable of evaluating the trade-offs between 
system economy and stability. Finally, in order to demonstrate potential applicability of the IFRTSRP model, it is applied to a hypothetical case study of water resources management.

\section{Materials and methods}

\section{Modeling formulation}

Consider a problem wherein water resources managers are responsible for allocating water from multiple unregulated surface and ground water sources to different users. The managers need to promise allowable targets of water supplies for each user, which can help the users to make their development plans. If the promised water can be delivered, a higher net benefit can be obtained; however, if the promised water can not be delivered, the net benefit will be reduced due to the penalty imposed. The water availabilities are randomly varied. When only a small chance of receiving sufficient water is predicted, the users will curb their development plans to avoid high penalties; however, the development opportunities will also be missed when the practical available water amounts are sufficient. Conversely, when the available water amounts are overestimated, the users will make major development plans; however, the penalty costs will also be increased when the practical available water amounts are insufficient. Thus, the related decisions must be made under varying probability levels, and a TSP model with recourse can solve the problem of water allocation.

However, water resources management systems are also filled with other uncertainties. For example, the water availabilities are hard to be precisely determined under each probability, and thus they are also expressed as fuzzy boundary intervals; the water allocation targets and the benefit and cost coefficients may not be available as deterministic values, and thus they are expressed as discrete intervals. Therefore, in order to reflect multiple uncertainties and achieve effective water allocation, based on the integration of TSP, FRP and ILP, an IFRTSP model can be formulated as follows:

$$
\begin{aligned}
\max f^{ \pm}= & \sum_{i=1}^{I} \sum_{m=1}^{M}\left(\mathrm{NB}_{i}^{ \pm}-\mathrm{TC}_{i m}^{ \pm}\right) W_{i m}^{ \pm} \\
& +\sum_{i=1}^{I} \sum_{n=1}^{N}\left(\mathrm{NB}_{i}^{ \pm}-\mathrm{TC}_{i n}^{ \pm}-\mathrm{TR}_{i n}^{ \pm}\right) W_{i n}^{ \pm} \\
& -\sum_{i=1}^{I} \sum_{m=1}^{M} \sum_{j=1}^{J} P_{m j} \mathrm{CW}_{i}^{ \pm} D_{i m j}^{ \pm} \\
& -\sum_{i=1}^{I} \sum_{n=1}^{N} \sum_{h=1}^{H} P_{n h} \mathrm{CW}_{i}^{ \pm} D_{i n h}^{ \pm}
\end{aligned}
$$

subject to:

$$
\begin{aligned}
& \sum_{i=1}^{I}\left(W_{i m}^{ \pm}-D_{i m j}^{ \pm}\right)\left(1+\tilde{\delta}_{i m}^{ \pm}\right) \leq \tilde{q}_{m j}^{ \pm}, \quad \forall m, j \\
& \sum_{i=1}^{I}\left(W_{i n}^{ \pm}-D_{i n h}^{ \pm}\right)\left(1+\tilde{\delta}_{i n}^{ \pm}\right) \leq \tilde{q}_{n h}^{ \pm}, \quad \forall n, h \\
& W_{i m \max }^{ \pm} \geq W_{i m}^{ \pm} \geq D_{i m j}^{ \pm} \geq 0, \quad \forall i, m, j \\
& W_{i n \max }^{ \pm} \geq W_{i n}^{ \pm} \geq D_{i n h}^{ \pm} \geq 0, \quad \forall i, n, h
\end{aligned}
$$

where $f^{ \pm}, \mathrm{NB}_{i}^{ \pm}, \mathrm{CW}_{i}^{ \pm}, \mathrm{TC}_{i m}^{ \pm}, \mathrm{TC}_{i n}^{ \pm}, \mathrm{TR}_{i n}^{ \pm}, D_{i m j}^{ \pm}, D_{i n h}^{ \pm}, W_{i m}^{ \pm}$, $W_{i n}^{ \pm}, W_{\text {im } \max }^{ \pm}$and $W_{\text {in } \max }^{ \pm}$denote the sets of discrete interval numbers; $\tilde{q}_{m j}^{ \pm}, \tilde{q}_{n h}^{ \pm}, \tilde{\delta}_{i m}^{ \pm}$and $\tilde{\delta}_{i n}^{ \pm}$denote the sets of fuzzy boundary interval numbers; $i$ is the water user; $m$ and $n$ are the surface and ground water sources, respectively; $j$ and $h$ are the levels of water availabilities from surface and ground water sources, respectively; $f^{ \pm}$is the net system benefit; $\mathrm{NB}_{i}^{ \pm}$are the benefits to user $i$ per unit of water allocated; $\mathrm{CW}_{i}^{ \pm}$are the penalty costs to user $i$ per unit of water not delivered; $\mathrm{TC}_{i m}^{ \pm}$and $\mathrm{TC}_{i n}^{ \pm}$are the delivering costs for allocating per unit of water from surface water source $m$ and ground water source $n$ to user $i$, respectively; $\mathbf{T R}_{i n}^{ \pm}$ are the costs for pumping per unit of water from ground water source $n$ to user $i ; P_{m j}$ are the probabilities of occurrences of water availability level $j$ from surface water source $m\left(\sum_{j=1}^{J} P_{m j}=1\right) ; P_{n h}$ are the probabilities of occurrences of water availability level $h$ from ground water source $n\left(\sum_{h=1}^{H} P_{n h}=1\right), \tilde{q}_{m j}^{ \pm}$are the water availabilities under level $j$ from surface water source $m ; \tilde{q}_{n h}^{ \pm}$ are the water availabilities under level $h$ from ground water source $n ; W_{i m}^{ \pm}$and $W_{i n}^{ \pm}$are the water allocation targets that are promised to user $i$ from surface water source $m$ and ground water source $n$, respectively; $W_{i m \max }^{ \pm}$ and $W_{\text {in } \max }^{ \pm}$are the maximum allowable allocation amounts for user $i$ from surface water source $m$ and ground water source $n$, respectively; $D_{i m j}^{ \pm}$are the amounts by which the respective water allocation targets are not met when the water availabilities are $\tilde{q}_{m j}^{ \pm}$with probabilities $P_{m j}$ from surface water source $m ; D_{i n h}^{ \pm}$are the amounts by which the respective water allocation targets are not met when the water availabilities are $\tilde{q}_{n h}^{ \pm}$with probabilities $P_{n h}$ from ground water source $n . \tilde{\delta}_{i m}^{ \pm}$and $\tilde{\delta}_{i n}^{ \pm}$ are the loss rates of water from surface water source $m$ and ground water source $n$ to user $i$ during transportation, respectively.

Furthermore, in water resources management systems, water allocation patterns should ensure both feasibility and reliability, even though such allocation patterns may lead to low net system benefits. Feasibility means the practicability of water allocation patterns and is related to water 
deficits; reliability represents the extent of a balanced consideration for the objective function values under various probability levels (Xu et al. 2009b). The higher the feasibility, the lower the water deficits; the higher the reliability, the lower the variability among the obtained expected values of objective functions.

In the IFRTSP model, since the objective function is the maximization of economic benefit, the economy-optimal solution can be obtained through determining high allocation targets and allocating a number of water to these users with high benefits; however, such water allocation can lead to high water deficits, especially in low water availability levels. Moreover, to minimize the expected values of penalty costs can hardly guarantee the minimization of penalty costs under various probability levels. In such conditions, the system stability (including system feasibility and system reliability) will be relatively low. SRO method can solve the problem by bringing risk concern into optimization models. Thus, SRO is incorporated within the IFRTSP framework, which leads to the IFRTSRP model for water resources management:

$$
\begin{aligned}
\max f^{ \pm}= & \sum_{i=1}^{I} \sum_{m=1}^{M}\left(\mathrm{NB}_{i}^{ \pm}-\mathrm{TC}_{i m}^{ \pm}\right) W_{i m}^{ \pm} \\
+ & \sum_{i=1}^{I} \sum_{n=1}^{N}\left(\mathrm{NB}_{i}^{ \pm}-\mathrm{TC}_{i n}^{ \pm}-\mathrm{TR}_{i n}^{ \pm}\right) W_{i n}^{ \pm} \\
& -\sum_{i=1}^{I} \sum_{m=1}^{M} \sum_{j=1}^{J} P_{m j} \mathrm{CW}_{i}^{ \pm} D_{i m j}^{ \pm}-\sum_{i=1}^{I} \sum_{n=1}^{N} \sum_{h=1}^{H} P_{n h} \mathrm{CW}_{i}^{ \pm} D_{i n h}^{ \pm} \\
& -\lambda \sum_{m=1}^{M} \sum_{j=1}^{J} P_{m j}\left|\sum_{i=1}^{I} \mathrm{CW}_{i}^{ \pm} D_{i m j}^{ \pm}-\sum_{i=1}^{I} \sum_{j=1}^{J} P_{m j} \mathrm{CW}_{i}^{ \pm} D_{i m j}^{ \pm}\right| \\
& -\omega \sum_{n=1}^{N} \sum_{h=1}^{H} P_{n h}\left|\sum_{i=1}^{I} \mathrm{CW}_{i}^{ \pm} D_{i n h}^{ \pm}-\sum_{i=1}^{I} \sum_{h=1}^{H} P_{n h} \mathrm{CW}_{i}^{ \pm} D_{i n h}^{ \pm}\right|
\end{aligned}
$$

subject to:

$$
\begin{aligned}
& \sum_{i=1}^{I}\left(W_{i m}^{ \pm}-D_{i m j}^{ \pm}\right)\left(1+\tilde{\delta}_{i m}^{ \pm}\right) \leq \tilde{q}_{m j}^{ \pm}, \quad \forall m, j \\
& \sum_{i=1}^{I}\left(W_{i n}^{ \pm}-D_{i n h}^{ \pm}\right)\left(1+\tilde{\delta}_{i n}^{ \pm}\right) \leq \tilde{q}_{n h}^{ \pm}, \quad \forall n, h \\
& W_{i m \max }^{ \pm} \geq W_{i m}^{ \pm} \geq D_{i m j}^{ \pm} \geq 0, \quad \forall i, m, j \\
& W_{i n \max }^{ \pm} \geq W_{i n}^{ \pm} \geq D_{i n h}^{ \pm} \geq 0, \quad \forall i, n, h
\end{aligned}
$$

where $\lambda$ and $\omega$ represent the non-negative weight coefficients. The terms of $\left|\sum_{i=1}^{I} \mathrm{CW}_{i}^{ \pm} D_{i m j}^{ \pm}-\sum_{i=1}^{I} \sum_{j=1}^{J} P_{m j} \mathrm{CW}_{i}^{ \pm} D_{i m j}^{ \pm}\right|$ and $\left|\sum_{i=1}^{I} \mathrm{CW}_{i}^{ \pm} D_{i n h}^{ \pm}-\sum_{i=1}^{I} \sum_{h=1}^{H} P_{n h} \mathrm{CW}_{i}^{ \pm} D_{i n h}^{ \pm}\right|$are the variability measures on the penalty costs. Depending on the values of $\lambda$ and $\omega$, the optimization may favor solutions with higher expected costs of $\sum_{i=1}^{I} \sum_{m=1}^{M} \sum_{j=1}^{J} P_{m j} \mathrm{CW}_{i}^{ \pm} D_{i m j}^{ \pm}$and $\sum_{i=1}^{I} \sum_{n=1}^{N} \sum_{h=1}^{H} P_{n h} \mathrm{CW}_{i}^{ \pm} D_{i n h}^{ \pm}$in exchange for the lower variability on the penalty costs as measured by $\left|\sum_{i=1}^{I} \mathrm{CW}_{i}^{ \pm} D_{i m j}^{ \pm}-\sum_{i=1}^{I} \sum_{j=1}^{J} P_{m j} \quad \mathrm{CW}_{i}^{ \pm} D_{i m j}^{ \pm}\right| \quad$ and $\quad \mid \sum_{i=1}^{I}$ $\mathrm{CW}_{i}^{ \pm} D_{i n h}^{ \pm}-\sum_{i=1}^{I} \sum_{h=1}^{H} P_{n h} \mathrm{CW}_{i}^{ \pm} D_{i n h}^{ \pm} \mid$, respectively (Takriti and Ahmed 2004). In order to solve model (2) through the linearization of $\left|\sum_{i=1}^{I} \mathrm{CW}_{i}^{ \pm} D_{i m j}^{ \pm}-\sum_{i=1}^{I} \sum_{j=1}^{J} P_{m j} \mathrm{CW}_{i}^{ \pm} D_{i m j}^{ \pm}\right|$ and $\left|\sum_{i=1}^{I} \mathrm{CW}_{i}^{ \pm} D_{i n h}^{ \pm}-\sum_{i=1}^{I} \sum_{h=1}^{H} P_{n h} \mathrm{CW}_{i}^{ \pm} D_{i n h}^{ \pm}\right|$, a goal programming method is incorporated within model (2) (Yu and $\operatorname{Li} 2000)$ :

$$
\begin{aligned}
\max f^{ \pm}= & \sum_{i=1}^{I} \sum_{m=1}^{M}\left(\mathrm{NB}_{i}^{ \pm}-\mathrm{TC}_{i m}^{ \pm}\right) W_{i m}^{ \pm} \\
& +\sum_{i=1}^{I} \sum_{n=1}^{N}\left(\mathrm{NB}_{i}^{ \pm}-\mathrm{TC}_{i n}^{ \pm}-\mathrm{TR}_{i n}^{ \pm}\right) W_{i n}^{ \pm} \\
& -\sum_{i=1}^{I} \sum_{m=1}^{M} \sum_{j=1}^{J} P_{m j} \mathrm{CW}_{i}^{ \pm} D_{i m j}^{ \pm}-\sum_{i=1}^{I} \sum_{n=1}^{N} \sum_{h=1}^{H} P_{n h} \mathrm{CW}_{i}^{ \pm} D_{i n h}^{ \pm} \\
& -\lambda \sum_{m=1}^{M} \sum_{j=1}^{J} P_{m j}\left(\sum_{i=1}^{I} \mathrm{CW}_{i}^{ \pm} D_{i m j}^{ \pm}-\sum_{i=1}^{I} \sum_{j=1}^{J} P_{m j} \mathrm{CW}_{i}^{ \pm} D_{i m j}^{ \pm}+2 \theta_{m j}^{ \pm}\right) \\
& -\omega \sum_{n=1}^{N} \sum_{h=1}^{H} P_{n h}\left(\sum_{i=1}^{I} \mathrm{CW}_{i}^{ \pm} D_{i n h}^{ \pm}-\sum_{i=1}^{I} \sum_{h=1}^{H} P_{n h} \mathrm{CW}_{i}^{ \pm} D_{i n h}^{ \pm}+2 \theta_{n h}^{ \pm}\right)
\end{aligned}
$$

subject to:

$$
\sum_{i=1}^{I} \mathrm{CW}_{i}^{ \pm} D_{i m j}^{ \pm}-\sum_{i=1}^{I} \sum_{j=1}^{J} P_{m j} \mathrm{CW}_{i}^{ \pm} D_{i m j}^{ \pm}+\theta_{m j}^{ \pm} \geq 0, \quad \forall m, j
$$$$
\sum_{i=1}^{I} \mathrm{CW}_{i}^{ \pm} D_{i n h}^{ \pm}-\sum_{i=1}^{I} \sum_{h=1}^{H} P_{n h} \mathrm{CW}_{i}^{ \pm} D_{i n h}^{ \pm}+\theta_{n h}^{ \pm} \geq 0, \quad \forall n, h
$$

$$
\sum_{i=1}^{I}\left(W_{i m}^{ \pm}-D_{i m j}^{ \pm}\right)\left(1+\tilde{\delta}_{i m}^{ \pm}\right) \leq \tilde{q}_{m j}^{ \pm}, \quad \forall m, j
$$

$$
\sum_{i=1}^{I}\left(W_{i n}^{ \pm}-D_{i n h}^{ \pm}\right)\left(1+\tilde{\delta}_{i n}^{ \pm}\right) \leq \tilde{q}_{n h}^{ \pm}, \quad \forall n, h
$$

$W_{i m \max }^{ \pm} \geq W_{i m}^{ \pm} \geq D_{i m j}^{ \pm} \geq 0, \quad \forall i, m, j$

$W_{\text {in } \max }^{ \pm} \geq W_{\text {in }}^{ \pm} \geq D_{\text {inh }}^{ \pm} \geq 0, \quad \forall i, n, h$

$\theta_{m j}^{ \pm} \geq 0, \quad \forall m, j$

$\theta_{n h}^{ \pm} \geq 0, \quad \forall n, h$ 
where $\theta_{m j}^{ \pm}$and $\theta_{n h}^{ \pm}$are the slack variables; constraints (3b) and (3c) are the specific control constraints. After the introduction of the variability measures in the objective function, model (3) can not only effectively reflect multiple uncertainties, but also guarantee solutions to be more stable and reliable.

To solve the IFRTSRP problem, assumptions are made in this solution process: For each parameter presented as fuzzy boundary interval, the fuzzy sets of the lower and upper bounds have no intersections and dependences (Nie et al. 2007). Based on the assumptions, when the water allocation targets $\left(W_{i m}^{ \pm}\right.$and $\left.W_{i n}^{ \pm}\right)$are known, the IFRTSRP problem can be solved within an ILP framework by utilizing FRP optimization techniques. Firstly, based on an interactive algorithm (Huang et al. 1992; Huang 1996), model (3) can be transformed into two sets of submodels, which correspond to the upper and lower bounds of the desired objective function value. Secondly, according to the concept of level set (fuzzy $\alpha$-cut) and the representation theorem (Negoita et al. 1976), each fuzzy constraint in the submodels can be replaced by $2 S$ precise inequalities, in which $S$ denotes the number of $\alpha$-cut levels (Soyster 1973; Leung 1988; Luhandjula and Gupta 1996; Liu et al. 2003). Finally, following these replacements, the decision spaces in the submodels can be delimited by the deterministic constraints, and then these two sets of submodels can be solved via simple method. The resulting interval solutions for the objective function and decision variables can easily interpret for generating decision alternatives.

In model (3), since the water allocation targets $\left(W_{i m}^{ \pm}\right.$and $\left.W_{i n}^{ \pm}\right)$are expressed as discrete interval numbers, decision variables $Z_{i m}$ and $Z_{i n}$ are introduced to determine the optimal allocation targets for supporting the related policy analyses (Huang and Loucks 2000). In detail, let $W_{i m}^{ \pm}=W_{i m}^{-}+Z_{i m} \Delta W_{i m}$, where $\Delta W_{i m}=W_{i m}^{+}-W_{i m}^{-}$and $Z_{\text {im }} \in[0,1] ; W_{\text {in }}^{ \pm}=W_{\text {in }}^{-}+Z_{\text {in }} \Delta W_{\text {in }}$, where $\Delta W_{\text {in }}=W_{\text {in }}^{+}-$ $W_{\text {in }}^{-}$and $Z_{\text {in }} \in[0,1]$. Thus, when $W_{i m}^{ \pm}$and $W_{\text {in }}^{ \pm}$approach their respective upper bounds (i.e., when $Z_{i m}=1$ and $Z_{\text {in }}=1$ ), a higher net system benefit would be achieved as long as the water demands are well satisfied; however, a higher penalty may have to be paid when the promised water is not delivered. Conversely, when $W_{i m}^{ \pm}$and $W_{i n}^{ \pm}$ reach their respective lower bounds (i.e., when $Z_{i m}=0$ and $Z_{i n}=0$ ), the system may have a lower net benefit with a lower risk of violating the promised water targets and a lower penalty. Therefore, it is difficult to determine whether $W_{i m}^{+}$or $W_{i m}^{-}$as well as $W_{i n}^{+}$or $W_{i n}^{-}$would correspond to the desired upper bound of net system benefit. Thus, according to the related studies (Negoita et al. 1976; Luhandjula and Gupta 1996; Huang and Loucks 2000; Liu et al. 2003; Nie et al. 2007), a two-step method associated with various $\alpha$-cut levels and the representation theorem can be used to solve model (3). In detail, the first submodel can be formulated as follows:

$$
\begin{aligned}
\max f^{+}= & \sum_{i=1}^{I} \sum_{m=1}^{M}\left(\mathrm{NB}_{i}^{+}-\mathrm{TC}_{i m}^{-}\right)\left(W_{i m}^{-}+Z_{i m} \Delta W_{i m}\right) \\
& +\sum_{i=1}^{I} \sum_{n=1}^{N}\left(\mathrm{NB}_{i}^{+}-\mathrm{TC}_{i n}^{-}-\mathrm{TR}_{i n}^{-}\right)\left(W_{i n}^{-}+Z_{i n} \Delta W_{i n}\right) \\
& -\sum_{i=1}^{I} \sum_{m=1}^{M} \sum_{j=1}^{J} P_{m j} \mathrm{CW}_{i}^{-} D_{i m j}^{-}-\sum_{i=1}^{I} \sum_{n=1}^{N} \sum_{h=1}^{H} P_{n h} \mathrm{CW}_{i}^{-} D_{i n h}^{-} \\
& -\lambda \sum_{m=1}^{M} \sum_{j=1}^{J} P_{m j}\left(\sum_{i=1}^{I} \mathrm{CW}_{i}^{-} D_{i m j}^{-}-\sum_{i=1}^{I} \sum_{j=1}^{J} P_{m j} \mathrm{CW}_{i}^{-} D_{i m j}^{-}+2 \theta_{m j}^{-}\right) \\
& -\omega \sum_{n=1}^{N} \sum_{h=1}^{H} P_{n h}\left(\sum_{i=1}^{I} \mathrm{CW}_{i}^{-} D_{i n h}^{-}-\sum_{i=1}^{I} \sum_{h=1}^{H} P_{n h} \mathrm{CW}_{i}^{-} D_{i n h}^{-}+2 \theta_{n h}^{-}\right)
\end{aligned}
$$

subject to:

$$
\begin{aligned}
& \sum_{i=1}^{I} \mathrm{CW}_{i}^{-} D_{i m j}^{-}-\sum_{i=1}^{I} \sum_{j=1}^{J} P_{m j} \mathrm{CW}_{i}^{-} D_{i m j}^{-}+\theta_{m j}^{-} \geq 0, \forall m, j \\
& \sum_{i=1}^{I} \mathrm{CW}_{i}^{-} D_{i n h}^{-}-\sum_{i=1}^{I} \sum_{h=1}^{H} P_{n h} \mathrm{CW}_{i}^{-} D_{i n h}^{-}+\theta_{n h}^{-} \geq 0, \forall n, h \\
& \sum_{i=1}^{I}\left(W_{i m}^{-}+Z_{i m} \Delta W_{i m}-D_{i m j}^{-}\right)\left(1+\overline{\delta_{i m}^{-s}}\right) \leq \overline{q_{m j}^{+}},
\end{aligned}
$$

$\forall m, j ; s=1,2, \ldots, S$

$\sum_{i=1}^{I}\left(W_{i m}^{-}+Z_{i m} \Delta W_{i m}-D_{i m j}^{-}\right)\left(1+\underline{\delta_{i m}^{-s}}\right) \geq \underline{q_{m j}^{+s}}$,

$\forall m, j ; s=1,2, \ldots, S$

$\sum_{i=1}^{I}\left(W_{i n}^{-}+Z_{i n} \Delta W_{i n}-D_{i n h}^{-}\right)\left(1+{\overline{\delta_{i n}^{-}}}^{s}\right) \leq \overline{{\overline{q_{n h}^{+}}}^{s}}$,

$\forall n, h ; s=1,2, \ldots, S$

$\sum_{i=1}^{I}\left(W_{i n}^{-}+Z_{i n} \Delta W_{i n}-D_{i n h}^{-}\right)\left(1+\underline{\delta_{i n}^{-s}}\right) \geq \underline{q_{n h}^{+s}}$,

$\forall n, h ; s=1,2, \ldots, S$

$W_{i m \text { max }}^{+} \geq W_{i m}^{-}+Z_{i m} \Delta W_{i m} \geq D_{i m j}^{-} \geq 0, \quad \forall i, m, j$

$W_{\text {in } \max }^{+} \geq W_{\text {in }}^{-}+Z_{\text {in }} \Delta W_{\text {in }} \geq D_{\text {inh }}^{-} \geq 0, \quad \forall i, n, h$

$\theta_{m j}^{-} \geq 0, \quad \forall m, j$

$\theta_{n h}^{-} \geq 0, \quad \forall n, h$

$0 \leq Z_{\text {im }} \leq 1, \quad \forall i, m$

$0 \leq Z_{\text {in }} \leq 1, \quad \forall i, n$

where the marks '- $s$ ' and ' $s$ ' denote the superior and inferior limits among the set $s ; Z_{i m}, Z_{i n}, D_{i m j}^{-}$, and $D_{i n h}^{-}$are decision variables. Solution for $f^{+}$provides the extreme upper bound of objective function under uncertain inputs. Let $f_{o p t}^{+}, Z_{i m \mathrm{opt}}, Z_{i n \mathrm{opt}}, D_{i m j \mathrm{opt}}^{-}$and $D_{\text {inh } \mathrm{opt}}^{-}$be solutions of 
submodel (4). Then, based on the solutions, the second submodel can be formulated as follows:

$$
\begin{aligned}
\max f^{-}= & \sum_{i=1}^{I} \sum_{m=1}^{M}\left(\mathrm{NB}_{i}^{-}-\mathrm{TC}_{i m}^{+}\right)\left(W_{i m}^{-}+Z_{\text {imopt }} \Delta W_{\text {im }}\right) \\
& +\sum_{i=1}^{I} \sum_{n=1}^{N}\left(\mathrm{NB}_{i}^{-}-\mathrm{TC}_{i n}^{+}-\mathrm{TR}_{\text {in }}^{+}\right)\left(W_{\text {in }}^{-}+Z_{\text {in opt }} \Delta W_{i n}\right) \\
& -\sum_{i=1}^{I} \sum_{m=1}^{M} \sum_{j=1}^{J} P_{m j} \mathrm{CW}_{i}^{+} D_{i m j}^{+}-\sum_{i=1}^{I} \sum_{n=1}^{N} \sum_{h=1}^{H} P_{n h} \mathrm{CW}_{i}^{+} D_{i n h}^{+} \\
& -\lambda \sum_{m=1}^{M} \sum_{j=1}^{J} P_{m j}\left(\sum_{i=1}^{I} \mathrm{CW}_{i}^{+} D_{i m j}^{+}-\sum_{i=1}^{I} \sum_{j=1}^{J} P_{m j} \mathrm{CW}_{i}^{+} D_{i m j}^{+}+2 \theta_{m j}^{+}\right) \\
& -\omega \sum_{n=1}^{N} \sum_{h=1}^{H} P_{n h}\left(\sum_{i=1}^{I} \mathrm{CW}_{i}^{+} D_{i n h}^{+}-\sum_{i=1}^{I} \sum_{h=1}^{H} P_{n h} \mathrm{CW}_{i}^{+} D_{i n h}^{+}+2 \theta_{n h}^{+}\right)
\end{aligned}
$$

subject to:

$$
\begin{aligned}
& \sum_{i=1}^{I} \mathrm{CW}_{i}^{+} D_{i m j}^{+}-\sum_{i=1}^{I} \sum_{j=1}^{J} P_{m j} \mathrm{CW}_{i}^{+} D_{i m j}^{+}+\theta_{m j}^{+} \geq 0, \forall m, j \\
& \sum_{i=1}^{I} \mathrm{CW}_{i}^{+} D_{i n h}^{+}-\sum_{i=1}^{I} \sum_{h=1}^{H} P_{n h} \mathrm{CW}_{i}^{+} D_{i n h}^{+}+\theta_{n h}^{+} \geq 0, \forall n, h \\
& \sum_{i=1}^{I}\left(W_{i m}^{-}+Z_{i m \text { opt }} \Delta W_{i m}-D_{i m j}^{+}\right)\left(1+\overline{\delta_{i m}^{+}}\right) \leq \overline{q_{m j}^{-}} s,
\end{aligned}
$$

$\forall m, j ; s=1,2, \ldots, S$

$\sum_{i=1}^{I}\left(W_{i m}^{-}+Z_{i m \text { opt }} \Delta W_{i m}-D_{i m j}^{+}\right)\left(1+\underline{\delta_{i m}^{+s}}\right) \geq \underline{q_{m j}^{-s}}$,

$\forall m, j ; s=1,2, \ldots, S$

$\sum_{i=1}^{I}\left(W_{i n}^{-}+Z_{\text {in opt }} \Delta W_{i n}-D_{i n h}^{+}\right)\left(1+\overline{\delta_{i n}^{+}}\right) \leq \overline{q_{n h}^{-}}$,

$\forall n, h ; s=1,2, \ldots, S$

$\sum_{i=1}^{I}\left(W_{i n}^{-}+Z_{\text {in opt }} \Delta W_{\text {in }}-D_{\text {inh }}^{+}\right)\left(1+\underline{\delta_{i n}^{+s}}\right) \geq \underline{q_{n h}^{-s}}$,

$\forall n, h ; s=1,2, \ldots, S$

$W_{i m \max }^{-} \geq W_{i m}^{-}+Z_{\text {im opt }} \Delta W_{i m} \geq D_{\text {imj }}^{+} \geq 0, \quad \forall i, m, j$

$W_{\text {in } \max }^{-} \geq W_{\text {in }}^{-}+Z_{\text {in opt }} \Delta W_{\text {in }} \geq D_{\text {inh }}^{+} \geq 0, \quad \forall i, n, h$

$D_{i m j}^{+} \geq D_{i m j \mathrm{opt}}^{-}, \quad \forall i, m, j$

$D_{i n h}^{+} \geq D_{i n h \mathrm{opt}}^{-}, \quad \forall i, n, h$

$\theta_{m j}^{+} \geq 0, \quad \forall m, j$

$\theta_{n h}^{+} \geq 0, \quad \forall n, h$

where $D_{i m j}^{+}$and $D_{i n h}^{+}$are decision variables. Let $f_{\text {opt }}^{-}, D_{i m j \text { opt }}^{+}$ and $D_{i n h \text { opt }}^{+}$be solutions of submodel (5). Model (3) is converted to two deterministic linear programming submodels. Thus, combining solutions of submodels (4) and (5), solutions for model (3) can be obtained as follows: $f_{\text {opt }}^{ \pm}=\left[f_{\text {opt }}^{-}, f_{\text {opt }}^{+}\right]$

$W_{i m \mathrm{opt}}^{ \pm}=W_{i m}^{-}+Z_{i m \mathrm{opt}} \Delta W_{i m}, \quad \forall i, m$

$W_{\text {in opt }}^{ \pm}=W_{\text {in }}^{-}+Z_{\text {in opt }} \Delta W_{\text {in }}, \quad \forall i, n$

$D_{i m j \text { opt }}^{ \pm}=\left[D_{i m j \text { opt }}^{-}, D_{i m j \text { opt }}^{+}\right], \quad \forall i, m, j$

$D_{\text {inh } \mathrm{opt}}^{ \pm}=\left[D_{\text {inh opt }}^{-}, D_{\text {inh opt }}^{+}\right], \quad \forall i, n, h$

where $W_{i m \text { opt }}^{ \pm}$and $W_{\text {in opt }}^{ \pm}$are the optimized water allocation targets; $D_{i m j \text { opt }}^{ \pm}$and $D_{i n h \text { opt }}^{ \pm}$are the optimized water deficits. Thus, the optimal water allocation schemes are:

$A_{i m j \mathrm{opt}}^{ \pm}=W_{i m \mathrm{opt}}^{ \pm}-D_{i m j \mathrm{opt}}^{ \pm}, \quad \forall i, m, j$

$A_{\text {inh opt }}^{ \pm}=W_{\text {in opt }}^{ \pm}-D_{\text {inh } \mathrm{opt}}^{ \pm}, \quad \forall i, n, h$

Figure 1 shows the schematic of the IFRTSRP model. It is based on four optimization techniques, namely TSP, FRP, ILP and SRO. Each technique has a unique contribution to enhancing the model's capability in dealing with system

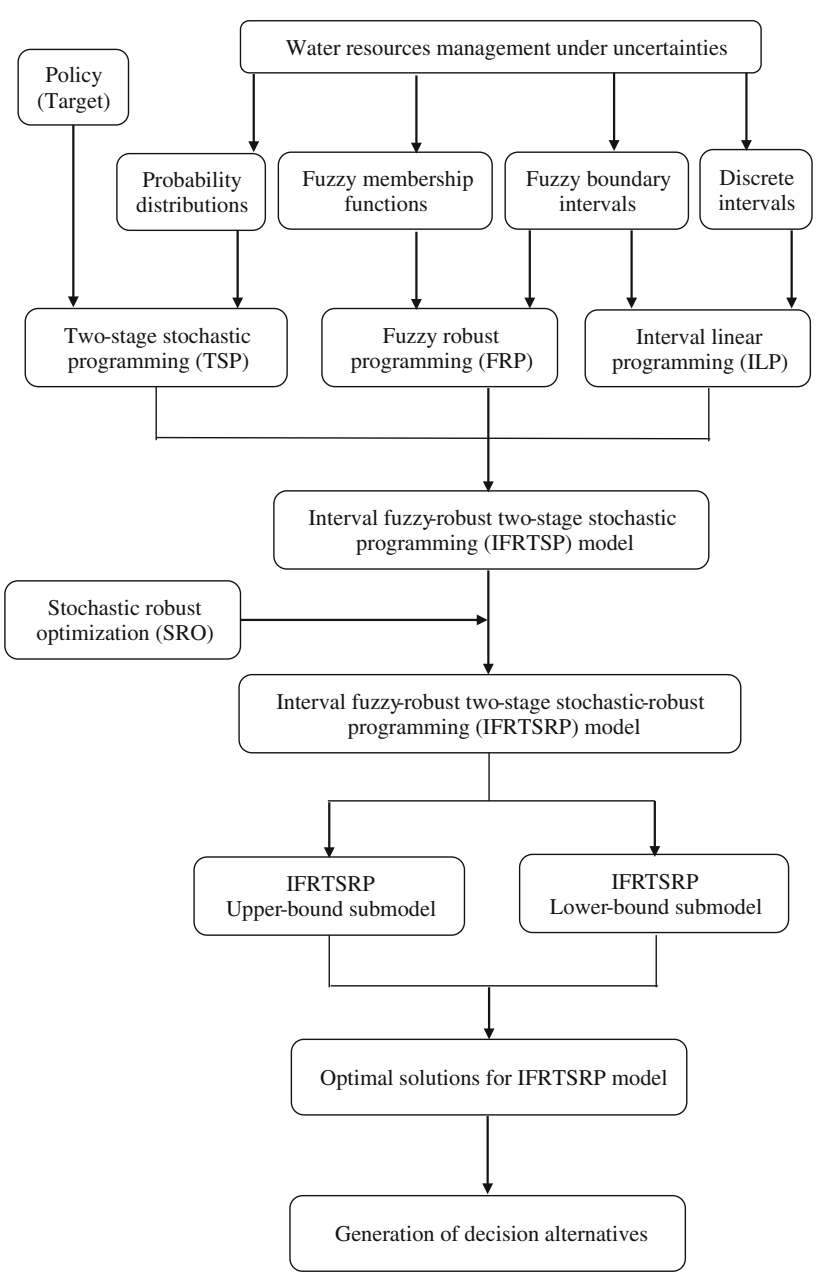

Fig. 1 Schematic of the IFRTSRP model 
Table 1 Benefits and related costs

\begin{tabular}{lccc}
\hline Activity & User & & \\
\cline { 2 - 4 } & User 1 & User 2 & User 3 \\
& $(i=1)$ & $(i=2)$ & $(i=3)$ \\
\hline Benefit when water is delivered & {$[93.6$,} & {$[74.4$,} & {$[58.3$,} \\
$\left(\$ / \mathrm{m}^{3}\right)$ & $99.7]$ & $81.7]$ & $65.8]$ \\
Delivering cost from surface water & {$[31.4$,} & {$[37.5$,} & {$[40.3$,} \\
source to users $\left(\$ / \mathrm{m}^{3}\right)$ & $34.9]$ & $41.7]$ & $43.7]$ \\
Delivering cost from ground water & {$[14.5$,} & {$[12.9$,} & {$[11.2$,} \\
source to users $\left(\$ / \mathrm{m}^{3}\right)$ & $16.3]$ & $15.5]$ & $13.8]$ \\
Pumping cost from ground water & {$[22.0$,} & {$[22.0$,} & {$[22.0$,} \\
source to users $\left(\$ / \mathrm{m}^{3}\right)$ & $25.0]$ & $25.0]$ & $25.0]$ \\
Penalty cost when water is not & {$[82.9$,} & {$[56.1$,} & {$[35.0$,} \\
delivered $\left(\$ / \mathrm{m}^{3}\right)$ & $89.2]$ & $61.5]$ & $39.5]$ \\
\hline
\end{tabular}

Table 2 Water allocation targets for each user

\begin{tabular}{llll}
\hline Water source & User & & \\
\cline { 2 - 4 } & $\begin{array}{l}\text { User 1 } \\
(i=1)\end{array}$ & $\begin{array}{l}\text { User } 2 \\
(i=2)\end{array}$ & $\begin{array}{l}\text { User 3 } \\
(i=3)\end{array}$ \\
\hline $\begin{array}{l}\text { Water allocation target }\left(10^{6} \mathrm{~m}^{3}\right) \\
\text { Surface water } \\
\text { source }\end{array}$ & {$[18.0,21.5]$} & {$[14.0,17.0]$} & {$[12.5,16.0]$} \\
$\begin{array}{l}\text { Ground water } \\
\text { source }\end{array}$ & {$[10.5,13.5]$} & {$[11.0,13.5]$} & {$[12.5,16.0]$} \\
$\begin{array}{l}\text { Maximum allowable allocation }\left(10^{6} \mathrm{~m}^{3}\right) \text { from different water sources } \\
\text { to users }\end{array}$ & {$[22.0,26.0]$} & {$[20.0,23.0]$} & {$[20.0,23.0]$} \\
$\begin{array}{l}\text { Surface water } \\
\text { source }\end{array}$ & {$[15.0,17.0]$} & {$[14.0,17.0]$} & {$[18.0,20.0]$} \\
$\begin{array}{l}\text { Ground water } \\
\text { source }\end{array}$ & & & \\
\hline
\end{tabular}

uncertainties and system risks. For example, the probability distributions and policy implications can be handled by TSP; the uncertainties expressed as fuzzy membership functions can be handled by FRP; the uncertainties expressed as discrete intervals can be handled by ILP; the uncertainties expressed as fuzzy boundary intervals can be handled by FRP within an ILP framework; the system risks can be addressed by SRO. Thus, the IFRTSRP model can not only tackle multiple uncertainties, but also provide an effective linkage between the pre-regulated water resources management policies and the associated economic implications. The IFRTSRP model can enhance the robustness for the optimization process by delimiting the uncertain decision space through dimensional enlargement of the original fuzzy constraints. The IFRTSRP model can also evaluate the trade-offs between system economy and stability. The IFRTSRP model will offer feasible and reliable solutions, and the interval solutions can provide two extreme scenarios, which are helpful for the water resources managers. Compared with the IFRTSP model, the IFRTSRP model can simultaneously take system economy and stability into consideration, and achieve a more stable water allocation pattern. Moreover, the solutions from the IFRTSRP model can provide opportunities for the managers to make water resources management policies and plans based on the reasonable consideration of system benefit, system stability and system failure risk.

Case study

The developed IFRTSRP model is applied to a hypothetical water resources management problem to demonstrate its

Table 3 Water availabilities under different $\alpha$-cut levels and associated probabilities from different water sources

\begin{tabular}{|c|c|c|c|c|c|c|}
\hline \multirow[t]{2}{*}{ Level of water availability } & \multirow[t]{2}{*}{ Probability } & \multicolumn{5}{|c|}{ Water availability $\left(10^{6} \mathrm{~m}^{3}\right)$} \\
\hline & & $\alpha=0$ & $\alpha=0.2$ & $\alpha=0.5$ & $\alpha=0.8$ & $\alpha=1$ \\
\hline \multicolumn{7}{|l|}{ Surface water source } \\
\hline Low $(j=1)$ & 0.2 & $\begin{array}{l}{[[24.0,27.0],} \\
[28.0,32.0]]\end{array}$ & $\begin{array}{l}{[[24.2,26.6],} \\
[28.2,31.4]]\end{array}$ & $\begin{array}{l}{[[24.5,26.0],} \\
[28.5,30.5]]\end{array}$ & $\begin{array}{l}{[[24.8,25.4],} \\
[28.8,29.6]]\end{array}$ & {$[25.0,29.0]$} \\
\hline Medium $(j=2)$ & 0.6 & $\begin{array}{l}{[[35.0,39.0],} \\
[40.0,44.0]]\end{array}$ & $\begin{array}{l}{[[35.2,38.4],} \\
[40.2,43.4]]\end{array}$ & $\begin{array}{l}{[[35.5,37.5],} \\
[40.5,42.5]]\end{array}$ & $\begin{array}{l}{[[35.8,36.6],} \\
[40.8,41.6]]\end{array}$ & {$[36.0,41.0]$} \\
\hline High $(j=3)$ & 0.2 & $\begin{array}{l}{[[48.0,52.0],} \\
[53.0,58.0]]\end{array}$ & $\begin{array}{l}{[[48.2,51.4],} \\
[53.2,57.2]]\end{array}$ & $\begin{array}{l}{[[48.5,50.5],} \\
[53.5,56.0]]\end{array}$ & $\begin{array}{l}{[[48.8,49.6],} \\
[53.8,54.8]]\end{array}$ & {$[49.0,54.0]$} \\
\hline \multicolumn{7}{|l|}{ Ground water source } \\
\hline Low $(h=1)$ & 0.1 & $\begin{array}{l}{[[16.00,18.00],} \\
[19.00,22.00]]\end{array}$ & $\begin{array}{l}{[[16.10,17.70],} \\
[19.10,21.50]]\end{array}$ & $\begin{array}{l}{[[16.25,17.25],} \\
[19.25,20.75]]\end{array}$ & $\begin{array}{l}{[[16.40,16.80],} \\
[19.40,20.00]]\end{array}$ & {$[16.50,19.50]$} \\
\hline Medium $(h=2)$ & 0.8 & $\begin{array}{l}{[[25.00,27.00],} \\
[28.00,32.00]]\end{array}$ & $\begin{array}{l}{[[25.10,26.70],} \\
[28.20,31.40]]\end{array}$ & $\begin{array}{l}{[[25.25,26.25],} \\
[28.50,30.50]]\end{array}$ & $\begin{array}{l}{[[25.40,25.80],} \\
[28.80,29.60]]\end{array}$ & {$[25.50,29.00]$} \\
\hline High $(h=3)$ & 0.1 & $\begin{array}{l}{[[33.50,36.00],} \\
[37.50,41.50]]\end{array}$ & $\begin{array}{l}{[[33.60,35.60],} \\
[37.70,40.90]]\end{array}$ & $\begin{array}{l}{[[33.75,35.00],} \\
[38.00,40.00]]\end{array}$ & $\begin{array}{l}{[[33.90,34.40],} \\
[38.30,39.10]]\end{array}$ & {$[34.00,38.50]$} \\
\hline
\end{tabular}


Table 4 Water loss rates under different $\alpha$-cut levels between different water sources and users

\begin{tabular}{|c|c|c|c|c|c|}
\hline \multirow[t]{2}{*}{ User } & \multicolumn{5}{|l|}{$\alpha$-Cut level } \\
\hline & $\alpha=0$ & $\alpha=0.2$ & $\alpha=0.5$ & $\alpha=0.8$ & $\alpha=1$ \\
\hline \multicolumn{6}{|c|}{ Loss rate for water transportation between surface water source and users } \\
\hline User $1(i=1)$ & $\begin{array}{l}{[[0.0600,0.0900],} \\
[0.1000,0.1400]]\end{array}$ & $\begin{array}{l}{[[0.0650,0.0890],} \\
[0.1060,0.1380]]\end{array}$ & $\begin{array}{l}{[[0.0725,0.0875],} \\
[0.1150,0.1350]]\end{array}$ & $\begin{array}{l}{[[0.0800,0.0860],} \\
[0.1240,0.1320]]\end{array}$ & {$[0.0850,0.1300]$} \\
\hline User $2(i=2)$ & $\begin{array}{l}{[[0.1300,0.1600],} \\
[0.1700,0.2200]]\end{array}$ & $\begin{array}{l}{[[0.1350,0.1590],} \\
[0.1780,0.2180]]\end{array}$ & $\begin{array}{l}{[[0.1425,0.1575],} \\
[0.1900,0.2150]]\end{array}$ & $\begin{array}{l}{[[0.1500,0.1560],} \\
[0.2020,0.2120]]\end{array}$ & {$[0.1550,0.2100]$} \\
\hline User $3(i=3)$ & $\begin{array}{l}{[[0.2000,0.2500],} \\
[0.2600,0.3000]]\end{array}$ & $\begin{array}{l}{[[0.2080,0.2480],} \\
[0.2670,0.2990]]\end{array}$ & $\begin{array}{l}{[[0.2200,0.2450],} \\
[0.2775,0.2975]]\end{array}$ & $\begin{array}{l}{[[0.2320,0.2420],} \\
[0.2880,0.2960]]\end{array}$ & {$[0.2400,0.2950]$} \\
\hline \multicolumn{6}{|c|}{ Loss rate for water transportation between ground water source and users } \\
\hline User $1(i=1)$ & $\begin{array}{l}{[[0.1200,0.1600],} \\
[0.1700,0.2000]]\end{array}$ & $\begin{array}{l}{[[0.1270,0.1590],} \\
[0.1750,0.1990]]\end{array}$ & $\begin{array}{l}{[[0.1375,0.1575],} \\
[0.1825,0.1975]]\end{array}$ & $\begin{array}{l}{[[0.1480,0.1560],} \\
[0.1900,0.1960]]\end{array}$ & {$[0.1550,0.1950]$} \\
\hline User $2(i=2)$ & $\begin{array}{l}{[[0.0600,0.0900],} \\
[0.1000,0.1300]]\end{array}$ & $\begin{array}{l}{[[0.0650,0.0890],} \\
[0.1050,0.1290]]\end{array}$ & $\begin{array}{l}{[[0.0725,0.0875],} \\
[0.1125,0.1275]]\end{array}$ & $\begin{array}{l}{[[0.0800,0.0860],} \\
[0.1200,0.1260]]\end{array}$ & {$[0.0850,0.1250]$} \\
\hline User $3(i=3)$ & $\begin{array}{l}{[[0.0200,0.0450],} \\
[0.0500,0.0800]]\end{array}$ & $\begin{array}{l}{[[0.0240,0.0440],} \\
[0.0550,0.0790]]\end{array}$ & $\begin{array}{l}{[[0.0300,0.0425],} \\
[0.0625,0.0775]]\end{array}$ & $\begin{array}{l}{[[0.0360,0.0410],} \\
[0.0700,0.0760]]\end{array}$ & {$[0.0400,0.0750]$} \\
\hline
\end{tabular}

applicability. The water resources managers are responsible for allocating water from two water sources (i.e., one surface water source and one ground water source) to three users. Each user wants to know how much water they can expect. If water supplies are insufficient, they will curb their development plans; conversely, if the promised water is delivered, they will make major development plans to achieve higher net system benefit. In the water resources management system, in addition to the pursuit in maximization of net system benefit, the system feasibility and reliability issues are taken into consideration. Therefore, the problems under consideration include: (1) analyzing how to effectively allocate water to the three users to achieve a maximum net system benefit with a stable water allocation policy under multiple uncertainties; and (2) analyzing how to achieve the feasibility and reliability of water allocation patterns, and evaluating the trade-offs between system economy and stability. To solve these problems, the developed IFRTSRP model is considered to be suitable.

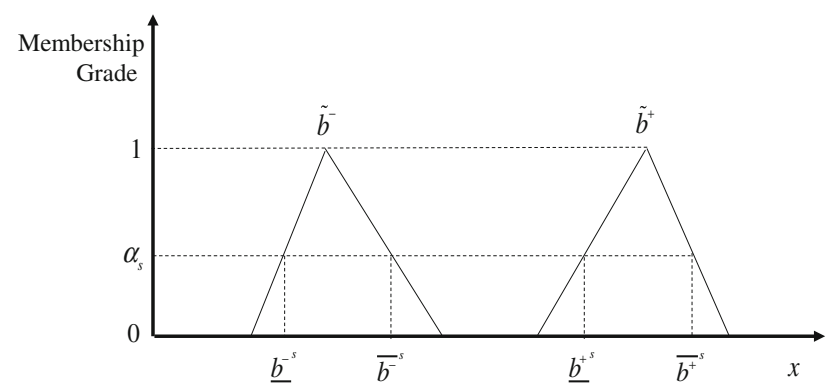

Fig. 2 Triangular fuzzy membership functions for fuzzy boundaries of $\tilde{b}^{ \pm}$
Table 1 shows the related economic data. Table 2 shows the water allocation targets for each user. All of the parameters are represented as discrete interval numbers (e.g., $a^{ \pm}$) to address the uncertainties: $a^{ \pm}=\left[a^{-}, a^{+}\right]=\left\{t \in a \mid a^{-} \leq t \leq a^{+}\right\}$, where $a^{-}$and $a^{+}$are the deterministic lower and upper bounds of $a^{ \pm}$. Table 3 lists the varied water availabilities as well as the associated probabilities of occurrences. Table 4 lists the water loss rates between different water sources and users. Water availabilities and water loss rates are represented as fuzzy boundary intervals, and left-right (L-R) fuzzy membership functions can be used to express fuzzy boundaries (Dubois and Prade 1978). In this study, the triangular fuzzy membership functions, which belong to $\mathbf{L}-\mathbf{R}$ fuzzy membership functions, are used to express fuzzy boundaries (Fig. 2), and the fuzzy boundary interval parameters (e.g., $\left.\tilde{b}^{ \pm}\right)$can be expressed: $\tilde{b}^{ \pm}=\left[\tilde{b}^{-}, \tilde{b}^{+}\right]=\left[\left[\underline{b^{-s}},{\overline{b^{-}}}^{s}\right],\left[\underline{b^{+s}}, \overline{b^{+}}{ }^{s}\right]\right]$.

\section{Results and discussion}

Results analysis

Table 5 presents the solutions of water allocation targets for users from different water sources under different $\lambda$ and $\omega$ levels. For example, when both $\lambda$ and $\omega$ levels are 0.5 , the optimized water allocation targets from surface water source for user 1 , user 2 , and user 3 would be $21.5 \times 10^{6}$ $\left(Z_{1 m}=1.0\right), \quad 14.0 \times 10^{6} \quad\left(Z_{2 m}=0\right), \quad$ and $12.5 \times 10^{6}$ $\left(Z_{3 m}=0\right) \mathrm{m}^{3}$, respectively; the optimized water allocation targets from ground water source for user 1, user 2, and user 3 would be $13.5 \times 10^{6}\left(Z_{1 n}=1.0\right), 11.0 \times 10^{6}$ $\left(Z_{2 n}=0\right)$, and $12.5 \times 10^{6}\left(Z_{3 n}=0\right) \mathrm{m}^{3}$, respectively. The 
results demonstrate that when $\lambda=0.5$ and $\omega=0.5$, the managers are optimistic of water supply to user 1 ; conversely, the managers have a conservative attitude toward water allocation to user 2 and user 3. Moreover, variations in water allocation targets can reflect different policies for water resources management under uncertainty. When the water allocation targets reach their upper bounds, the

Table 5 Solutions of water allocation targets from different water sources under different $\lambda$ and $\omega$ levels

\begin{tabular}{|c|c|c|c|}
\hline \multirow[t]{2}{*}{ User } & \multicolumn{3}{|c|}{ Optimized water allocation target $\left(10^{6} \mathrm{~m}^{3}\right)$} \\
\hline & $\begin{array}{l}\lambda=0.5 \\
\omega=0.5\end{array}$ & $\begin{array}{l}\lambda=1.5 \\
\omega=1.5\end{array}$ & $\begin{array}{l}\lambda=4 \\
\omega=4\end{array}$ \\
\hline \multicolumn{4}{|c|}{ Surface water source } \\
\hline User $1(i=1)$ & 21.5 & 21.3 & 18.0 \\
\hline User $2(i=2)$ & 14.0 & 14.0 & 14.0 \\
\hline User $3(i=3)$ & 12.5 & 12.5 & 15.1 \\
\hline \multicolumn{4}{|c|}{ Ground water source } \\
\hline User $1(i=1)$ & 13.5 & 12.2 & 11.7 \\
\hline User $2(i=2)$ & 11.0 & 11.0 & 11.0 \\
\hline User $3(i=3)$ & 12.5 & 12.5 & 12.5 \\
\hline
\end{tabular}

corresponding policy may result in a higher net system benefit but, at the same time, a higher risk of penalty when the water availability levels are low; conversely, when the water allocation targets approach their lower bounds, the corresponding policy may result in less water deficits as well as lower penalty costs but, at the same time, more waste of water resources when the water availability levels are medium or high. Thus, different policies in predefining the promised water allocation accounts are associated with different levels of system benefit and system failure risk.

Solutions in Table 5 also indicate that the optimized water allocation targets would vary with the changes of $\lambda$ and $\omega$ levels. For example, when both $\lambda$ and $\omega$ levels vary from 0.5 to 4 , the total optimized water allocation targets for all users from surface water source would change from $48.0 \times 10^{6}$ to $47.1 \times 10^{6} \mathrm{~m}^{3}$, and the total optimized water allocation targets for all users from ground water source would change from $37.0 \times 10^{6}$ to $35.2 \times 10^{6} \mathrm{~m}^{3}$. Generally, with the increase of $\lambda$ and $\omega$ levels, the total optimized water allocation targets for all users would decrease, which can reduce the total water deficits. Thus, with the increase of $\lambda$ and $\omega$ levels, the system failure risk would be lessened and the system feasibility would be

Table 6 Solutions of water deficits and water allocation amounts from different water sources under different $\lambda$ and $\omega$ levels

\begin{tabular}{|c|c|c|c|c|c|c|c|c|}
\hline \multirow[t]{2}{*}{ User } & \multirow[t]{2}{*}{$\begin{array}{l}\text { Level of water } \\
\text { availability }\end{array}$} & \multirow[t]{2}{*}{ Probability } & \multicolumn{2}{|l|}{$\begin{array}{l}\lambda=0.5 \\
\omega=0.5\end{array}$} & \multicolumn{2}{|l|}{$\begin{array}{l}\lambda=1.5 \\
\omega=1.5\end{array}$} & \multicolumn{2}{|l|}{$\begin{array}{l}\lambda=4 \\
\omega=4\end{array}$} \\
\hline & & & $\begin{array}{l}\text { Deficit } \\
\left(10^{6} \mathrm{~m}^{3}\right)\end{array}$ & $\begin{array}{l}\text { Allocation } \\
\left(10^{6} \mathrm{~m}^{3}\right)\end{array}$ & $\begin{array}{l}\text { Deficit } \\
\left(10^{6} \mathrm{~m}^{3}\right)\end{array}$ & $\begin{array}{l}\text { Allocation } \\
\left(10^{6} \mathrm{~m}^{3}\right)\end{array}$ & $\begin{array}{l}\text { Deficit } \\
\left(10^{6} \mathrm{~m}^{3}\right)\end{array}$ & $\begin{array}{l}\text { Allocation } \\
\left(10^{6} \mathrm{~m}^{3}\right)\end{array}$ \\
\hline \multicolumn{9}{|c|}{ Surface water source } \\
\hline \multirow{3}{*}{$\begin{array}{l}\text { User } 1 \\
\qquad(i=1)\end{array}$} & Low & 0.2 & 0 & 21.5 & 0 & 21.3 & 0 & 18.0 \\
\hline & Medium & 0.6 & 0 & 21.5 & 0 & 21.3 & 0 & 18.0 \\
\hline & High & 0.2 & 0 & 21.5 & 0 & 21.3 & 0 & 18.0 \\
\hline \multirow{3}{*}{$\begin{array}{l}\text { User } 2 \\
\qquad(i=2)\end{array}$} & Low & 0.2 & {$[8.6,13.1]$} & {$[0.9,5.4]$} & {$[8.4,12.9]$} & {$[1.1,5.6]$} & {$[5.3,9.9]$} & {$[4.1,8.7]$} \\
\hline & Medium & 0.6 & {$[0,3.9]$} & {$[10.1,14.0]$} & {$[0,3.7]$} & {$[10.3,14.0]$} & {$[0,0.6]$} & {$[13.4,14.0]$} \\
\hline & High & 0.2 & 0 & 14.0 & 0 & 14.0 & 0 & 14.0 \\
\hline \multirow{3}{*}{$\begin{array}{l}\text { User } 3 \\
\qquad(i=3)\end{array}$} & Low & 0.2 & 12.5 & 0 & 12.5 & 0 & 15.1 & 0 \\
\hline & Medium & 0.6 & {$[10.8,12.5]$} & {$[0,1.7]$} & {$[10.6,12.5]$} & {$[0,1.9]$} & {$[10.4,15.1]$} & {$[0,4.7]$} \\
\hline & High & 0.2 & {$[0.2,6.1]$} & {$[6.4,12.3]$} & {$[0,5.9]$} & {$[6.6,12.5]$} & {$[0,5.6]$} & {$[9.5,15.1]$} \\
\hline \multicolumn{9}{|c|}{ Ground water source } \\
\hline \multirow{3}{*}{$\begin{array}{l}\text { User } 1 \\
\qquad(i=1)\end{array}$} & Low & 0.1 & 0 & 13.5 & 0 & 12.2 & 0 & 11.7 \\
\hline & Medium & 0.8 & 0 & 13.5 & 0 & 12.2 & 0 & 11.7 \\
\hline & High & 0.1 & 0 & 13.5 & 0 & 12.2 & 0 & 11.7 \\
\hline \multirow{3}{*}{$\begin{array}{l}\text { User } 2 \\
\qquad(i=2)\end{array}$} & Low & 0.1 & {$[7.0,10.4]$} & {$[0.6,4.0]$} & {$[5.6,9.1]$} & {$[1.9,5.4]$} & {$[5.1,8.5]$} & {$[2.5,5.9]$} \\
\hline & Medium & 0.8 & {$[0,2.4]$} & {$[8.6,11.0]$} & {$[0,1.1]$} & {$[9.9,11.0]$} & {$[0,0.5]$} & {$[10.5,11.0]$} \\
\hline & High & 0.1 & 0 & 11.0 & 0 & 11.0 & 0 & 11.0 \\
\hline \multirow{3}{*}{$\begin{array}{l}\text { User } 3 \\
\qquad(i=3)\end{array}$} & Low & 0.1 & 12.5 & 0 & 12.5 & 0 & 12.5 & 0 \\
\hline & Medium & 0.8 & {$[10.5,12.5]$} & {$[0,2.0]$} & {$[9.1,12.5]$} & {$[0,3.4]$} & {$[8.6,12.5]$} & {$[0,3.9]$} \\
\hline & High & 0.1 & {$[1.4,7.0]$} & {$[5.5,11.1]$} & {$[0,5.6]$} & {$[6.9,12.5]$} & {$[0,5.1]$} & {$[7.4,12.5]$} \\
\hline
\end{tabular}


enhanced; conversely, lower $\lambda$ and $\omega$ levels would result in a higher system failure risk and lower system feasibility.

Table 6 presents the solutions of water deficits and water allocation amounts for each user from different water sources under different $\lambda$ and $\omega$ levels. The values of water deficits under given water allocation targets can reflect the variations of system conditions caused by uncertain inputs. Under advantageous conditions (e.g., when the other users do not consume the full amounts of the targeted demands and/or the water availabilities approach their fuzzy upper bounds), the water shortage levels may be low; however, under demanding conditions, the water deficits may be raised. For user 2 , when both $\lambda$ and $\omega$ levels are 0.5 , the optimized water deficits from surface water source would be $[8.6,13.1] \times 10^{6} \mathrm{~m}^{3}$ under low water availability level, $[0,3.9] \times 10^{6} \mathrm{~m}^{3}$ under medium water availability level, and $0 \mathrm{~m}^{3}$ under high water availability level; the optimized water deficits from ground water source would be [7.0, $10.4] \times 10^{6} \mathrm{~m}^{3}$ under low water availability level, $[0$, 2.4] $\times 10^{6} \mathrm{~m}^{3}$ under medium water availability level, and $0 \mathrm{~m}^{3}$ under high water availability level. The results indicate that, for user 2 , when both $\lambda$ and $\omega$ levels are 0.5 , under low water availability level, some water deficits would exist; under medium water availability level, the situation is more ambiguous: there may be no water deficits under advantageous conditions, and the water deficits may become higher under demanding conditions; under high water availability level, there would be no water deficits. The solutions for the other users and fixed $\lambda$ and $\omega$ levels can be similarly interpreted. Moreover, compared with user 1 and user 2, user 3 would generate higher water deficits. For example, when water availability levels are medium, and both $\lambda$ and $\omega$ levels are 0.5 , the optimized water deficits from surface water source for user 1, user 2 , and user 3 would be $0,[0,3.9] \times 10^{6}$, and $[10.8,12.5] \times 10^{6} \mathrm{~m}^{3}$, respectively; the optimized water deficits from ground water source for user 1 , user 2 , and user 3 would be 0 , $[0$, $2.4] \times 10^{6}$, and $[10.5,12.5] \times 10^{6} \mathrm{~m}^{3}$, respectively. This is because user 3 will obtain the lowest net benefits (i.e., $[14.6,25.5] \$ / \mathrm{m}^{3}$ from surface water source and [19.5, 32.6] $\$ / \mathrm{m}^{3}$ from ground water source) when the water demands are satisfied and encounter the lowest penalty costs if the promised water is not delivered. Thus, faced to the insufficient water supplies, the available water from surface and ground water sources is allocated first to user 1 , second to user 2 , and then to user 3.

Solutions in Table 6 also indicate that the optimized water deficits would vary with the changes of $\lambda$ and $\omega$ levels. For example, when the water availability levels are low, and both $\lambda$ and $\omega$ levels are $0.5,1.5$, and 4 , the total optimized water deficits for all users from surface water source would be $[21.1,25.6] \times 10^{6},[20.9,25.4] \times 10^{6}$, and $[20.4,25.0] \times 10^{6} \mathrm{~m}^{3}$, respectively; the total optimized water deficits for all users from ground water source would be $[19.5,22.9] \times 10^{6}$, [18.1, 21.6] $\times 10^{6}$, and [17.6, $21.0] \times 10^{6} \mathrm{~m}^{3}$, respectively. The variability measures are incorporated in the objective function, and their impacts on modeling outputs would be adjusted by the changes of $\lambda$ and $\omega$ levels. Generally, with the increase of $\lambda$ and $\omega$ levels, the total optimized water deficits for all users would decrease, which can reduce the system failure risk and enhance the system feasibility.

Solutions in Tables 5 and 6 indicate that when the water availability levels are low (the worst case condition), and both $\lambda$ and $\omega$ levels are 0.5 , the total water allocation amounts for all users from surface and ground water sources would be $[22.4,26.9] \times 10^{6}$ and $[14.1,17.5] \times 10^{6} \mathrm{~m}^{3}$, respectively; however, the total water demands from surface and ground water sources are $48.0 \times 10^{6}$ and $37.0 \times 10^{6} \mathrm{~m}^{3}$, respectively, which demonstrate that there are serious shortages in water supplies from surface and ground water sources. Although the probabilities of the worst case condition are low, the penalty costs from the occurrence of such an extreme event are high. In comparison, when the water availability levels are medium (the medium case condition), both and $\lambda$ and $\omega$ levels are 0.5 , the total water allocation amounts for all users from surface and ground water sources would be $[31.6,37.2] \times 10^{6}$ and $[22.1,26.5] \times 10^{6} \mathrm{~m}^{3}$, respectively; however, the total water demands from surface and ground water sources are $48.0 \times 10^{6}$ and $37.0 \times 10^{6}$ $\mathrm{m}^{3}$, respectively, which demonstrate that the water shortages are less serious than those under the worst case condition. When the water availability levels are high (the best case condition), and both $\lambda$ and $\omega$ levels are 0.5 , the total water allocation amounts for all users from surface and ground water sources would be $[41.9,47.8] \times 10^{6}$ and [30.0, $35.6] \times 10^{6} \mathrm{~m}^{3}$, respectively; however, the total water demands from surface and ground water sources are $48.0 \times 10^{6}$ and $37.0 \times 10^{6} \mathrm{~m}^{3}$, respectively, which demonstrate that the water deficits are further reduced and the water demands may basically be satisfied.

Table 7 shows the solutions of variability of penalty costs and net system benefits under different $\lambda$ and/or $\omega$ levels. The results indicate that the variability of penalty costs would gradually decrease with the increase of $\lambda$ and $\omega$ levels. For example, the variability (v), where $v=$ $\sum_{m=1}^{M} \sum_{j=1}^{J} P_{m j}\left(\sum_{i=1}^{I} \mathrm{CW}_{i}^{ \pm} D_{i m j}^{ \pm}-\sum_{i=1}^{I} \sum_{j=1}^{J} P_{m j} \mathrm{CW}_{i}^{ \pm} D_{i m j}^{ \pm}\right.$ $\left.+2 \theta_{m j}^{ \pm}\right)+\sum_{n=1}^{N} \sum_{h=1}^{H} P_{n h}\left(\sum_{i=1}^{I} \mathrm{CW}_{i}^{ \pm} D_{i n h}^{ \pm}-\sum_{i=1}^{I} \sum_{h=1}^{H}\right.$ $\left.P_{n h} \mathrm{CW}_{i}^{ \pm} D_{i n h}^{ \pm}+2 \theta_{n h}^{ \pm}\right)$would be $[668.9,969.5] \times 10^{6} \$$ under $\lambda=0.5$ and $\omega=0.5$, [638.4, 936.1] $\times 10^{6} \$$ under $\lambda=1.5$ and $\omega=1.5,[621.8,915.5] \times 10^{6} \$$ under $\lambda=2$ and $\omega=4$, and $[591.2,883.9] \times 10^{6} \$$ under $\lambda=4$ and $\omega=4$. Moreover, the intervals of the variability values 
Table 7 Solutions of variability of penalty costs and net system benefits under different $\lambda$ and/or $\omega$ levels

\begin{tabular}{lcccccccc}
\hline Activity & \multicolumn{1}{l}{$\lambda$ and $\omega$ levels } \\
\cline { 2 - 8 } & $\lambda=0.5$ & $\lambda=1.5$ & $\lambda=2$ & $\lambda=4$ & $\lambda=0.5$ & $\lambda=0.5$ & $\lambda=1.5$ & $\lambda=4$ \\
& $\omega=0.5$ & $\omega=1.5$ & $\omega=4$ & $\omega=4$ & $\omega=1.5$ & $\omega=4$ & $\omega=0.5$ & $\omega=0.5$ \\
\hline Variability $\left(10^{6} \$\right)$ & {$[668.9$,} & {$[638.4$,} & {$[621.8$,} & {$[591.2$,} & {$[645.0$,} & {$[636.9$,} & {$[662.3$,} & {$[623.1$,} \\
& $969.5]$ & $936.1]$ & $915.5]$ & $883.9]$ & $943.3]$ & $932.9]$ & $962.3]$ & $920.5]$ \\
Net system benefit & {$[1,821.5$,} & {$[1,833.9$,} & {$[1,837.4$,} & {$[1,755.9$,} & {$[1,835.3$,} & {$[1,840.7$,} & {$[1,820.1$,} & {$[1,736.7$,} \\
$\left(10^{6} \$\right)$ & $3,386.0]$ & $3,349.9]$ & $3,323.5]$ & $3,201.8]$ & $3,357.8]$ & $3,344.7]$ & $3,378.1]$ & $3,243.1]$ \\
\hline
\end{tabular}

would become narrow with the increase of $\lambda$ and $\omega$ levels. For example, the intervals would be $300.6 \times 10^{6} \$$ under $\lambda=0.5$ and $\omega=0.5,297.7 \times 10^{6} \$$ under $\lambda=1.5$ and $\omega=1.5,293.7 \times 10^{6} \$$ under $\lambda=2$ and $\omega=4$, and $292.7 \times 10^{6} \$$ under $\lambda=4$ and $\omega=4$. The varying trends of the variability of penalty costs imply that the system reliability would be enhanced with the increase of $\lambda$ and $\omega$ levels.

As shown in Table 7, all of the net system benefits are intervals. In practice, given different water availability conditions and underlying probability distributions, the resulting plans of optimized net system benefit would vary between its relevant solution interval. The plan with a higher net system benefit would correspond to a lower water shortage level under advantageous conditions, which can lead to a higher risk of the system failure; the plan with a lower net system benefit would better resist water shortage under demand conditions, which can lead to a lower risk of the system failure. The results also indicate that the optimized net system benefit could decrease as $\lambda$ and $\omega$ levels increase. For example, the optimized net system benefits would be $[1,821.5,3,386.0] \times 10^{6} \$$ under $\lambda=0.5$ and $\omega=0.5$, $[1,833.9,3,349.9] \times 10^{6} \$$ under $\lambda=1.5$ and $\omega=1.5$, $[1,837.4,3,323.5] \times 10^{6} \$$ under $\lambda=2$ and $\omega=4$, and $[1,755.9,3,201.8] \times 10^{6} \$$ under $\lambda=4$ and $\omega=4$. Moreover, the intervals of the net system benefit values would narrow down as $\lambda$ and $\omega$ levels increase. For example, the intervals would be $1,564.5 \times 10^{6} \$$ under $\lambda=0.5$ and $\omega=0.5,1,516.0 \times 10^{6} \$$ under $\lambda=1.5$ and $\omega=1.5,1,486.1 \times 10^{6} \$$ under $\lambda=2$ and $\omega=4$, and $1,445.9 \times 10^{6} \$$ under $\lambda=4$ and $\omega=4$. The varying trends of the net system benefit imply that a lower net system benefit could guarantee higher system stability; conversely, a higher net system benefit would correspond to lower system stability and higher system failure risk. Thus, the managers should make a choice between more stable solutions with a lower net system benefit and more variable solutions with a higher net system benefit, and a trade-off between system economy and stability can be used to help them to make costeffective decisions.

\section{Discussion}

In water resources management systems, for surface water sources and ground water sources, the water supplies are independent and the water allocation targets need to be respectively determined. Thus, in model (3), the objective function can be decomposed into: (1) $\max f_{1}^{ \pm}=$ $\sum_{i=1}^{I} \sum_{m=1}^{M}\left(\mathrm{NB}_{i}^{ \pm}-\mathrm{TC}_{i m}^{ \pm}\right) W_{i m}^{ \pm}-\sum_{i=1}^{I} \sum_{m=1}^{M} \sum_{j=1}^{J} P_{m j}$ $\mathrm{CW}_{i}^{ \pm} D_{i m j}^{ \pm}-\quad \lambda \sum_{m=1}^{M} \quad \sum_{j=1}^{J} P_{m j}\left(\sum_{i=1}^{I} \mathrm{CW}_{i}^{ \pm} D_{i m j}^{ \pm}-\sum_{i=1}^{I}\right.$ $\sum_{j=1}^{J} P_{m j} \mathrm{CW}_{i}^{ \pm} D_{i m j}^{ \pm}+2 \theta_{m j}^{ \pm}$) (the benefit of water supplies from surface water sources), and (2) $\max f_{2}^{ \pm}=$ $\sum_{i=1}^{I} \sum_{n=1}^{N}\left(\mathrm{NB}_{i}^{ \pm}-\mathrm{TC}_{i n}^{ \pm}-\mathrm{TR}_{i n}^{ \pm}\right) W_{i n}^{ \pm}-\sum_{i=1}^{I} \sum_{n=1}^{N} \sum_{h=1}^{H}$ $P_{n h} \mathrm{CW}_{i}^{ \pm} D_{i n h}^{ \pm}-w \sum_{n=1}^{N} \sum_{h=1}^{H} P_{n h}\left(\sum_{i=1}^{I} \mathrm{CW}_{i}^{ \pm} D_{i n h}^{ \pm}-\sum_{i=1}^{I}\right.$ $\sum_{h=1}^{H} P_{n h} \mathrm{CW}_{i}^{ \pm} D_{i n h}^{ \pm}+2 \theta_{n h}^{ \pm}$) (the benefit of water supplies from ground water sources); moreover, constraints (3b), (3d), (3f) and (3h) are the relevant constraints of water supplies from surface water sources, and constraints (3c), (3e), (3g) and (3i) are the relevant constraints of water supplies from ground water sources; furthermore, $\lambda$ and $\omega$ levels have no intersections, and their values affect the determination of water allocation targets from surface and ground water sources, respectively. Therefore, for all users, when $\omega$ level is any values, the total optimized water allocation targets and the total optimized water deficits from surface water sources would decrease with the increase of $\lambda$ level (Tables 5 and 6). Similarly, for all users, when $\lambda$ level is any values, the total optimized water allocation targets and the total optimized water deficits from ground water sources would decrease with the increase of $\omega$ level (Tables 5 and 6). Moreover, with the increase of $\lambda$ or $\omega$ levels, the variability of penalty costs and the optimized net system benefit would gradually decrease, and the intervals of these values would become narrow (Table 7). For example, when fixed $\lambda$ level is 0.5 , the variability of penalty costs would be $[668.9,969.5] \times 10^{6} \$$ under $\omega=0.5,[645.0,943.3] \times 10^{6} \$$ under $\omega=1.5$, and [636.9, 932.9] $\times 10^{6} \$$ under $\omega=4$, and their intervals would be $300.6 \times 10^{6} \$(\omega=0.5), 298.3 \times 10^{6} \$(\omega=1.5)$, and $296.0 \times 10^{6} \$(\omega=4)$. When fixed $\omega$ level is 0.5 , the optimized net system benefit would be $[1,821.5,3,386.0] \times 10^{6} \$$ under $\lambda=0.5,[1,820.1,3,378.1] \times 10^{6} \$$ under $\lambda=1.5$, and 
Table 8 Solutions of the IFRTSP model

\begin{tabular}{|c|c|c|c|c|c|}
\hline \multirow[t]{2}{*}{ User } & \multirow{2}{*}{$\begin{array}{l}\text { Level of water } \\
\text { availability }\end{array}$} & \multirow[t]{2}{*}{ Probability } & \multicolumn{3}{|c|}{ Optimized solution $\left(10^{6} \mathrm{~m}^{3}\right)$} \\
\hline & & & Target & Deficit & Allocation \\
\hline \multicolumn{6}{|c|}{ Surface water source } \\
\hline \multirow[t]{3}{*}{ User $1(i=1)$} & Low & 0.2 & 21.5 & 0 & 21.5 \\
\hline & Medium & 0.6 & 21.5 & 0 & 21.5 \\
\hline & High & 0.2 & 21.5 & 0 & 21.5 \\
\hline \multirow[t]{3}{*}{ User $2(i=2)$} & Low & 0.2 & 15.8 & {$[10.4,14.9]$} & {$[0.9,5.4]$} \\
\hline & Medium & 0.6 & 15.8 & {$[0,5.7]$} & {$[10.1,15.8]$} \\
\hline & High & 0.2 & 15.8 & 0 & 15.8 \\
\hline \multirow[t]{3}{*}{ User $3(i=3)$} & Low & 0.2 & 12.5 & 12.5 & 0 \\
\hline & Medium & 0.6 & 12.5 & 12.5 & 0 \\
\hline & High & 0.2 & 12.5 & {$[1.9,7.8]$} & {$[4.7,10.6]$} \\
\hline \multicolumn{6}{|c|}{ Ground water source } \\
\hline \multirow[t]{3}{*}{ User $1(i=1)$} & Low & 0.1 & 13.5 & 0 & 13.5 \\
\hline & Medium & 0.8 & 13.5 & 0 & 13.5 \\
\hline & High & 0.1 & 13.5 & 0 & 13.5 \\
\hline \multirow[t]{3}{*}{ User $2(i=2)$} & Low & 0.1 & 12.9 & {$[8.8,12.3]$} & {$[0.6,4.1]$} \\
\hline & Medium & 0.8 & 12.9 & {$[0,4.3]$} & {$[8.6,12.9]$} \\
\hline & High & 0.1 & 12.9 & 0 & 12.9 \\
\hline \multirow[t]{3}{*}{ User $3(i=3)$} & Low & 0.1 & 12.5 & 12.5 & 0 \\
\hline & Medium & 0.8 & 12.5 & 12.5 & 0 \\
\hline & High & 0.1 & 12.5 & {$[3.4,9.0]$} & {$[3.5,9.1]$} \\
\hline \multicolumn{2}{|c|}{ Net system benefit $\left(10^{6} \$\right)$} & {$[1,730.5,3$,} & & & \\
\hline
\end{tabular}

$[1,736.7,3,243.1] \times 10^{6} \$$ under $\lambda=4$, and their intervals would be $1,564.5 \times 10^{6} \$(\lambda=0.5), 1,558.0 \times 10^{6} \$$ $(\lambda=1.5)$, and $1,506.4 \times 10^{6} \$(\lambda=4)$. Thus, in practice, the managers can identify desired water allocation policies (i.e., surface water and/or ground water allocation policies) based on the choice of $\lambda$ or $\omega$ levels.

The water resources management problem is also solved by the IFRTSP model, and the solutions are shown in Table 8 . The results indicate that, compared with the solutions obtained by the IFRTSRP model, higher total water allocation targets for all users (i.e., $49.8 \times 10^{6} \mathrm{~m}^{3}$ from surface water source and $38.9 \times 10^{6} \mathrm{~m}^{3}$ from ground water source) would be generated, which could lead to higher total water deficits. For example, when the water availability levels are low, the total water deficits for all users from surface and ground water sources would be [22.9, $27.4] \times 10^{6}$ and $[21.3,24.8] \times 10^{6} \mathrm{~m}^{3}$, respectively; when the water availability levels are medium, the total water deficits for all users from surface and ground water sources would be $[12.5,18.2] \times 10^{6}$ and $[12.5,16.8] \times 10^{6} \mathrm{~m}^{3}$, respectively. Moreover, a higher net system benefit (i.e., $[1,730.5,3,414.1] \times 10^{6} \$$ ) would be obtained by the IFRTSP model compared with the IFRTSRP model. However, the IFRTSP model has some limitations. Firstly, the higher total water deficits would reduce the system feasibility and increase the system failure risk. Secondly, although the expected values of penalty costs are minimum, higher penalty costs would have to be paid under low water availability levels, which would reduce the system reliability. Finally, the solution intervals would be wider, which indicates that the system reliability would be worse.

In water resources management systems, the developed IFRTSRP model has also potential research extensions. Firstly, for a multi-period management problem, the IFRTSRP model is also suitable to solve it; however, the IFRTSRP model can hardly adequately reflect the dynamic variations of system conditions, especially for sequential structure of a large-scale problem (Li et al. 2006b). In fact, the water surpluses in the former period could be accumulated in the later period; nevertheless, the IFRTSRP model can not reflect such a variation, which leads to the application of multi-stage stochastic programming models. Secondly, the arbitrary variability measures are applied to reflect the variability of penalty costs, which could lead to non-optimal solutions and misleading decisions to the recourse problems (Takriti and Ahmed 2004); thus, the other methods in reflecting the variability should be investigated and applied.

The developed IFRTSRP model integrates different methods and is applied for water resources management under uncertainty. The model and the methods can provide 
a simple and effective management tool for water resources managers. Although the IFRTSRP model is only for water resources management problems, it is also of value for other environment management problems. Moreover, the IFRTSRP model can also combine with other inexact optimization methods to deal with various types of uncertainties, which can maximize net system benefits, achieve system stability, and reinforce problems decision support. Besides, the multi-criteria decision analysis technique can be used for further supporting the adjustments of modeling results. Furthermore, the intelligent decision support system can be developed based on an integration of optimization modeling, scenario development, user interaction, policy analysis, and visual display.

\section{Conclusion}

In this study, an IFRTSRP model has been developed for water resources management under uncertainty. The developed IFRTSRP model incorporates two-stage stochastic programming (TSP), fuzzy robust programming (FRP), interval linear programming (ILP), and stochastic robust optimization (SRO) within a general optimization framework, and can effectively deal with multiple uncertainties presented as probability distributions, fuzzy membership functions, discrete interval numbers, and their combinations. The IFRTSRP model can provide an effective linkage between the pre-regulated water resources management policies and the associated economic implications. By delimiting the uncertain decision space through dimensional enlargement of the original fuzzy constraints, the IFRTSRP model can enhance the robustness for the optimization process. Moreover, the variability measures are applied to reflect the variability of penalty costs, and the IFRTSRP model can evaluate the trade-offs between system economy and stability. The obtained solutions are the combinations of deterministic, interval and distributional information, and the interval solutions can help the managers to obtain multiple decision alternatives.

The developed IFRTSRP model has been applied to a hypothetical case study of water resources management. The obtained solutions have been analyzed for generating decision alternatives under different $\lambda$ and/or $\omega$ levels and various system conditions. The results indicate that a higher net system benefit would correspond to lower system stability; conversely, a lower net system benefit would guarantee higher system stability. The modeling results can help the managers to generate desired water allocation policies based on the reasonable consideration of net system benefit and system stability. Although application of IFRTSRP model for water resources management is a new attempt and the model could be further enhanced or extended, the modeling results imply that the model is applicable and effective in water allocation through the trade-offs among system economy, system stability, and system failure risk.

Acknowledgments This research was supported by the Environmental Protection Public Welfare Scientific Research Project (201309063), and the Fundamental Research Funds for the Central Universities (JB2011025). The authors would like to express thanks to the editor and the anonymous reviewers for their constructive comments and suggestions.

\section{References}

Amin MT, Alazba AA, EINesr MN (2013) Adaptation of climate variability/extreme in arid environment of the Arabian peninsula by rainwater harvesting and management. Int $\mathbf{J}$ Environ Sci Technol 10(1):27-36

Cai YP, Huang GH, Lu HW, Yang ZF, Tan Q (2009) I-VFRP: an interval-valued fuzzy robust programming approach for municipal waste-management planning under uncertainty. Eng Optim 41(5):399-418

Chang NB, Wen CG, Chen YL, Yong YC (1996) A grey fuzzy multiobjective programming approach for the optimal planning of a reservoir watershed. Part A: theoretical development. Water Res 30(10):2329-2334

Chen C, Huang GH, Li YP, Zhou Y (2013) A robust risk analysis method for water resources allocation under uncertainty. Stoch Environ Res Risk Assess 27(3):713-723

Chenini I, Ben Mammou A, Turki MM (2008) Groundwater resources of a multi-layered aquiferous system in arid area: data analysis and water budgeting. Int J Environ Sci Technol 5(3):361-374

Dubois D, Prade H (1978) Operations on fuzzy numbers. Int J Syst Sci 9(6):613-626

Dupačová J (1998) Reflections on robust optimization. Stochastic programming methods and technical applications. Springer, Berlin, pp 111-127

Feiring BR, Sastri T, Sim LSM (1998) A stochastic programming model for water resource planning. Math Comput Model 27(3):1-7

Gaivoronski AA, Sechi GM, Zuddas P (2012) Balancing cost-risk in management optimization of water resource systems under uncertainty. Phys Chem Earth 42-44:98-107

Guo P, Huang GH (2009) Two-stage fuzzy chance-constrained programming: application to water resources management under dual uncertainties. Stoch Environ Res Risk Assess 23(3):349-359

Guo P, Huang GH, Zhu H, Wang XL (2010) A two-stage programming approach for water resources management under randomness and fuzziness. Environ Model Softw 25(12):1573-1581

Han Y, Huang YF, Wang GQ (2011) Interval-parameter linear optimization model with stochastic vertices for land and water resources allocation under dual uncertainty. Environ Eng Sci 28(3):197-205

Han M, Ren QW, Wang Y, Du J, Hao Z, Sun FL, Cheng L, Qi SZ, Li DQ (2013) Integrated approach to water allocation in river basins. J Water Resour Plan Manag ASCE 139(2):159-165

Hoppe H, Weilandt M, Orth H (2004) A combined water management approach based on river water quality standards. J Environ Inform 3(2):67-76

Hu Q, Huang GH, Liu ZF, Fan YR, Li W (2012) Inexact fuzzy twostage programming for water resources management in an 
environment of fuzziness and randomness. Stoch Environ Res Risk Assess 26(2):261-280

Huang GH (1996) IPWM: an interval parameter water quality management model. Eng Optim 26(2):79-103

Huang GH, Loucks DP (2000) An inexact two-stage stochastic programming model for water resources management under uncertainty. Civ Eng Environ Syst 17(2):95-118

Huang GH, Baetz BW, Patry GG (1992) A grey linear programming approach for municipal solid waste management planning under uncertainty. Civ Eng Syst 9(4):319-335

Jairaj PG, Vedula S (2000) Multireservoir system optimization using fuzzy mathematical programming. Water Resour Manag 14(6):457-472

Kara SS, Onut S (2010) A stochastic optimization approach for paper recycling reverse logistics network design under uncertainty. Int J Environ Sci Technol 7(4):717-730

Karamouz M, Kerachian R, Zahraie B (2004) Monthly water resources and irrigation planning: case study of conjunctive use of surface and groundwater resources. J Irrig Drain Eng ASCE 130(5):391-402

Leung Y (1988) Spatial analysis and planning under imprecision (studies in regional science and urban economics). Elsevier Science Publishers, Amsterdam

Leung SCH, Tsang SOS, Ng WL, Wu Y (2007) A robust optimization model for multi-site production planning problem in an uncertain environment. Eur J Oper Res 181(1):224-238

Li YP, Huang GH (2009) Two-stage planning for sustainable waterquality management under uncertainty. J Environ Manag 90(8):2402-2413

Li YP, Huang GH, Nie SL (2006a) An interval-parameter multi-stage stochastic programming model for water resources management under uncertainty. Adv Water Resour 29(5):776-789

Li YP, Huang GH, Nie SL, Huang YF (2006b) IFTSIP: interval fuzzy two-stage stochastic mixed-integer linear programming: A case study for environmental management and planning. Civ Eng Environ Syst 23(2):73-99

Li YP, Huang GH, Nie SL (2009) A robust interval-based minimaxregret analysis approach for the identification of optimal waterresources-allocation strategies under uncertainty. Resour Conserv Recycl 54(2):86-96

Liu L, Huang GH, Liu Y, Fuller GA, Zeng GM (2003) A fuzzystochastic robust programming model for regional air quality management under uncertainty. Eng Optim 35(2):177-199

Liu M, Huang GH, Liao RF, Li YP, Xie YL (2013) Fuzzy two-stage non-point source pollution management model for agricultural systems - a cases study for the Lake Tai Basin, China. Agric Water Manag 121:27-41

Lu HW, Huang GH, He L (2009) Inexact rough-interval two-stage stochastic programming for conjunctive water allocation problems. J Environ Manag 91(9):261-269

Luhandjula MK, Gupta MM (1996) On fuzzy stochastic optimization. Fuzzy Sets Syst 81(1):47-55

Maqsood I, Huang GH (2003) A two-stage interval-stochastic programming model for waste management under uncertainty. J Air Waste Manag Assoc 53(5):540-552

Maqsood M, Huang GH, Yeomans JS (2005) An interval-parameter fuzzy two-stage stochastic program for water resources management under uncertainty. Eur J Oper Res 167(1):208-225
Mulvey JM, Ruszczynski A (1995) A new scenario decomposition method for large-scale stochastic optimization. Oper Res 43(3):477-490

Mulvey JM, Vanderbei RJ, Zenios SA (1995) Robust optimization of large-scale systems. Oper Res 43(2):264-281

Negoita CV, Minoiu S, Stan E (1976) On considering imprecision in dynamic linear programming. Econ Comput Econ Cybern Stud Res 3:83-95

Nie XH, Huang GH, Li YP, Liu L (2007) IFRP: a hybrid intervalparameter fuzzy robust programming approach for waste management planning under uncertainty. J Environ Manag 84(1):1-11

Noyan N (2012) Risk-averse two-stage stochastic programming with an application to disaster management. Comput Oper Res 39(3):541-559

Olsen D, Dickson SE, Baetz B (2006) Decision support system for rural water supply in the Nilgris district of South India. J Environ Inform 7(1):1-13

Sadegh M, Mahjouri N, Kerachian R (2010) Optimal inter-basin water allocation using crisp and fuzzy Shapley games. Water Resour Manag 24(10):2291-2310

Sethi LN, Panda SN, Nayak MK (2006) Optimal crop planning and water resources allocation in a coastal groundwater basin, Orissa. India. Agric Water Manag 83(3):209-220

Shao LG, Qin XS, Xu Y (2011) A conditional value-at-risk based inexact water allocation model. Water Resour Manag 25(9):2125-2145

Singh L, Dhillon JS (2008) Fuzzy satisfying multiobjective thermal power dispatch based on surrogate worth trade-off method. Electr Power Compon Syst 36(1):93-108

Soyster AL (1973) Technical note-convex programming with setinclusive constraints and applications to inexact linear programming. Oper Res 21(5):1154-1157

Takriti S, Ahmed S (2004) On robust optimization of two-stage systems. Math Program 99(1):109-126

Tan RR, Cruz DE (2004) Synthesis of robust water reuse networks for single-component retrofit problems using symmetric fuzzy linear programming. Comput Chem Eng 28(12):2547-2551

Trezos T, Yeh WWG (1987) Use of stochastic dynamic programming for reservoir management. Water Resour Res 23(6):983-996

Wang BJ, Lee LL, Chen CH, Chang CN, Chao AC (2005) Optimization of semiconductor processing water management strategy. J Environ Inform 5(2):81-88

Watkins Jr DW, Mckinney DC (1997) Finding robust solutions to water resource problems. J Water Resour Plan Manag ASCE 123(1):49-58

Xu Y, Huang GH, Qin XS (2009a) Inexact two-stage stochastic robust optimization model for water resources management under uncertainty. Environ Eng Sci 26(12):1765-1776

Xu Y, Huang GH, Qin XS, Cao MF (2009b) SRCCP: a stochastic robust chance-constrained programming model for municipal solid waste management under uncertainty. Resour Conserv Recycl 53(6):352-363

Yu CS, Li HL (2000) A robust optimization model for stochastic logistic problems. Int J Prod Econ 64(1-3):385-397 